\title{
Editorial:
}

\section{Special issue on distributed computing and artificial intelligence}

Juan M. CORCHADO ${ }^{1}$, Li WEIGANG ${ }^{2}$, Javier BAJO ${ }^{3}$, Fei WU ${ }^{4}$, Tian-cheng $\mathrm{LI}^{\dagger 1}$

('University of Salamanca, Spain)

$\left({ }^{2}\right.$ University of Brasilia, Brazil)

( ${ }^{3}$ Polytechnic University of Madrid, Spain)

$\left({ }^{4}\right.$ Zhejiang University, China)

†Email: t.c.li@usal.es

http://dx.doi.org/10.1631/FITEE.DCAI2015

4:1! Google's artificial intelligence (AI) program, AlphaGo, has won Go Master Lee Sedol in a best-of-five competition held in Korean March 9-15, 2016. Seen by many as a landmark moment for AI, the outcome did not come as a surprise, considering the excellent combination of 1920 CPUs with sophisticated AI algorithms, including neural networks and Monte Carlo tree search (Gibney, 2016; Silver et al., 2016). Indeed, research on distributed computing and artificial intelligence (DCAI) has matured during the last decade and many effective applications are now deployed, performing an increasingly important role in modern computer science, including the two most hyped technologies: Internet of Things and Big Data. Indeed, it is fair to say that the application of artificial intelligence in distributed environments is becoming an essential element of high added value and economic potential.

As a testimony to the considerable momentum in R\&D activities regarding DCAI, this special issue contains six outstanding papers from the 12th International Conference on DCAI and 13th International Conference on Practical Applications of Agents and Multi-Agent Systems held in the University of Salamanca, Spain, June 2015. The selected contributions cover new theories, techniques, and approaches on DCAI systems, from distributed adaptive searching to group decision making, from multi-agent system for crisis management to multi-robot scheduling for flow

(D) ORCID: Tian-cheng LI, http://orcid.org/0000-0002-0499-5135 (C) Zhejiang University and Springer-Verlag Berlin Heidelberg 2016 shop, and from ambient intelligence for entertainment to multi-camera monitoring systems for rehabilitation therapy.

The first paper by Wall (2016) investigates the effects of altering the organizational dynamics/setting of distributed adaptive search (DAS) processes, with emphasis on the complexity of interactions between partial search problems assigned to search agents. DAS processes occur in a large variety of real-world systems in which networked agents collaboratively search for higher levels of performance, obtaining collective intelligence, for which one scientific challenge is to coordinate and allow distributed agents to deal with the cross-agent interactions. The presented work is interesting because to reduce the problem complexity in many practical DAS applications, it is necessary to segment the overall search problem into disjoint partial problems, and then delegate them to different search agents. In such cases, dependencies across the agents' partial search problems become inevitable, which will cause significant performance degradation if not properly taken into account.

The second paper by Carneiro et al. (2016) presents an intelligent negotiation model to support the group decision making process, which facilitates arguments, complex algorithms, and agent modeling. In general, supporting group decision making in ubiquitous contexts is a complex task, as it has to deal with a large number of factors. Aware of the drawbacks of existing models that are barely adaptable to the business world reality, the presented work makes use of a social networking logic which simultaneously preserves the amount and quality of intelligence generated in face-to-face meetings, while defining strategies to deal with important points such as the type of attributes in multi-criterion problems, agents reasoning, and intelligent dialogues.

The third paper by de Brito et al. (2016) presents the use of situated artificial institution (SAI) within a hybrid, interactive, normative multi-agent system to regulate human collaboration in crisis management. 
To provide a context-aware crisis regulation, this paper introduces a constitutive level between environmental and normative states, providing a loose coupling of normative regulation with environment evolution, thus making it possible to keep both levels independent and easy-to-change in the face of complex and changing crisis situations. Norms are specified to regulate the actions of human actors based on both status functions and the actors' actions, leading to a declarative and distinct SAI modeling that manages the crisis with a context-aware crisis regulation.

The fourth paper by Rincon et al. (2016) involves a human-agent society where virtual agents and humans coexist and interact transparently into a fully integrated environment. This paper presents an ambient intelligence application where humans are immersed into a system and are treated as agents with an emotional state. What is particularly interesting is the resulting social emotional model which is able to maximize the welfare of humans by always playing the most appropriate music, in which each individual will be represented by an agent that has an emotional response according to its musical taste. The varying emotions of each agent are then collected and used to update the social emotion of the group. The emotional state of agents is indicated as one important issue to consider in the human-agent society.

The fifth paper by Nielsen et al. (2016) presents a constraint propagation driven approach for multirobot task allocation in flow shop scheduling, providing a prompt service to a set of routine direct and reverse queries. This involves a resourceconstrained multi-product scheduling problem for an automated guided vehicle (AGV) served flow shop, where multiple material handling transport modes provide movement of work pieces between machining centers in the multimodal transportation network (MTN). This network of repetitively acting local transportation modes encompassing an MTN structure provides a framework for multimodal process scheduling treated in terms of optimizing AGV fleet scheduling problems, subject to fuzzy operation time constraints and uncertainty of robots. In the presented work, both production takt and operations execution time are described by imprecise data.

In contrast to the above theoretical and/or novel approach study, the last paper by Oliver et al. (2016) stands out more from an experimental perspective, which investigates in detail how the overlap of several infrared beams affects the tracked position of the user, depending on the angle of incidence of light, distance to the target, distance between sensors, and the number of capture devices used. The experiment is carried out based on the Kinect camera, which probably represents one of the latest advances in cameras and three-dimensional capturing technologies. The experimental findings have enlightening significance for the design of intelligent patient-rehabilitation environments. This implies a good R\&D direction for using AI technologies to improve people's lives.

We would like to thank all the authors for their contribution to this special issue. We appreciate the dedication from the reviewers for their time and detailed reviews. It is our hope that these papers capture some of the latest major scientific developments and that they can serve as a springboard for further improvements and developments. The great support from the editorial office is also highly appreciated.

\section{References}

Carneiro, J., Martinho, D., Marreiros, G., et al., 2016. Intelligent negotiation model for ubiquitous group decision scenarios. Front. Inform. Technol. Electron. Eng., 17(4): 296-308. http://dx.doi.org/10.1631/FITEE.1500344

de Brito, M., Thévin, L., Garbay, C., et al., 2016. Supporting flexible regulation of crisis management by means of situated artificial institution. Front. Inform. Technol. Electron. Eng., 17(4):309-324. http://dx.doi.org/10.1631/FITEE.1500369

Gibney, E., 2016. Google AI algorithm masters ancient game of Go. Nature, 529:445-446. http://dx.doi.org/10.1038/529445a

Nielsen, I., Wójcik, R., Bocewicz, G., et al., 2016. Multimodal processes optimization subject to fuzzy operation time constraints: declarative modeling approach. Front. Inform. Technol. Electron. Eng., 17(4):338-347. http://dx.doi.org/10.1631/FITEE.1500359

Oliver, M., Montero, F., Molina, J.P., et al., 2016. Multicamera systems for rehabilitation therapies: a study of the precision of Microsoft Kinect sensors. Front. Inform. Technol. Electron. Eng., 17(4):348-364. http://dx.doi.org/10.1631/FITEE.1500347

Rincon, J.A., Bajo, J., Fernandez, A., et al., 2016. Using emotions for the development of human-agent societies. Front. Inform. Technol. Electron. Eng., 17(4):325-337. http://dx.doi.org/10.1631/FITEE.1500343

Silver, D., Huang, A., Maddison, C.J., et al., 2016. Mastering the game of Go with deep neural networks and tree search. Nature, 529:484-489. http://dx.doi.org/10.1038/nature16961

Wall, F., 2016. Organizational dynamics in adaptive distributed search processes: effects on performance and the role of complexity. Front. Inform. Technol. Electron. Eng., 17(4):283-295. http://dx.doi.org/10.1631/FITEE.1500306 